Short Technical Report

\title{
Multichannel Plating Unit for High-Throughput Plating of Cell Cultures
}

BioTechniques 33:420-423 (August 2002)

\author{
C.M. Hamilton, M. Anderson, \\ J. Lape, E. Creech ${ }^{1}$, and J. \\ Woessner \\ Paradigm Genetics, Research \\ Triangle Park, NC, and ${ }^{1}$ Current \\ Enterprises, Morrisville, NC, \\ USA
}

\section{INTRODUCTION}

The cloning of DNA fragments is usually the starting point for any sequence-based genomics study. To obtain representative or complete sequence of a genome of interest, genomic and cDNA libraries are constructed and are typically introduced into $E$. coli for clone propagation and subsequent sequence analysis. This traditionally labor-intensive, highthroughput cloning approach is now greatly facilitated by robotics systems that process the clones, including picking single colonies into 96- or 384-well culture plates, and prepare DNA for analysis $(3,4)$. However, more directed projects may require the cloning of specific genes of interest in a highthroughput mode. To achieve this, many processes required for cloning such as PCR, restriction enzyme digestion, and ligation have been adapted to the 96-well format. In addition, chemical transformation of E. coli can be carried out in a 96-well format (2). Even so, it is still necessary to plate out each of the transformations individually. This is typically accomplished by spreading each of the cultures onto its own agar plate to isolate individual colonies that correspond to each of the 96 clones. This process is labor intensive and requires 96 individual agar plates per 96-well plate.

Currently, the most commonly used method of plating out bacteria is to use an inoculating turntable (VWR International, West Chester, PA, USA) and a bacterial cell spreader. The spreader must be sterilized after each use, typically by dipping in ethanol and then flam- ing off the ethanol, a time-consuming and hazardous step. Recently, BD Biosciences Clontech (Palo Alto, CA, USA) developed CLONdisc ${ }^{\mathrm{TM}}$, a ready-made, wafer-thin culture system. Substituting the CLONdisc plates for conventional agar plates can speed up the plating process and reduces the storage and incubator space needed for traditional agar plates. However, each culture must be spread, one at a time, onto a corresponding CLONdisc plate. In a high-throughput environment, this process is potentially error prone and is expensive.

High-throughput genomic approaches to gene function or target identification have led to the development and implementation of the 96-well format for many standard molecular biology manipulations. The apparatus described here, a Multichannel Plating Unit, is designed to plate out individual cultures efficiently from standard 96-well culture blocks (1). This apparatus has 12 individual lanes, and the spacing is compatible with a standard 12-channel pipettor. Thus, the unit allows for the rapid plating of 12 individual cultures at a time. For one 96-well block of transformants, this method reduces the labeling and plating effort from 96 culture dishes that are spread individually to eight multichannel plates.

\section{MATERIALS AND METHODS}

\section{Materials}

With the exception of the lid and the glass beads, all parts were milled out of optically clear polycarbonate on a computer-controlled mill. The final 
products are opaque because of the milling process. Although polycarbonate is autoclavable, repeated autoclaving may cause warping and brittleness and will reduce the lifetime of the unit. Alternative methods of sterilization, such as washing with $70 \%$ ethanol, may be sufficient for many applications and may extend the lifetime of units made out of polycarbonate. Alternatively, materials that are more resistant to repeated autoclaving (e.g., polyphenylsulfone or polysulfone) may be used to construct the unit.

\section{Description of the Multichannel Plating Unit}

The components of the Multichannel Plating Unit are shown in Figure 1. The total width (internal dimension) of the unit (approximately $10.8 \mathrm{~cm}$ ) and the lane spacing (approximately $0.9 \mathrm{~cm}$ ) of the unit correspond to a standard 96well microplate. The length of the unit shown here is $20 \mathrm{~cm}$. The surface area of the agar in each lane is approximately $25 \%$ of a standard $(100 \mathrm{~mm})$ petri dish. There are 11 dividers, creating 12 lanes that extend $(19 \mathrm{~cm})$ to the exit alley. The dividers create a barrier at and above the surface of the agar and, in the unit shown here, are raised up off of the bottom of the unit (via underlying cross-supports) to facilitate filling the unit with agar. There is a loose-fitting lid so that the unit can be covered and inverted. Sterile glass beads spread the bacterial culture on the agar lanes. The beads are added to the unit using sterile tweezers and are placed between the start gate and the near edge of the unit. The start gate holds the sterile glass beads in place before and during the culture loading process. There is an exit block that defines an exit alley that facilitates the removal of the beads. The exit block is secured at the end of the unit before the agar media is poured and then removed after the media has solidified. There is a hole in one corner of the exit alley large enough to allow for the disposal of the beads into a biohazard container. The construction schematics of the unit are detailed at http://www.BioTechniques.com/figures/ aug02/hamilton construction.html.

\section{Preparation of the Multichannel Plating Unit}

All parts of the unit must be steril-

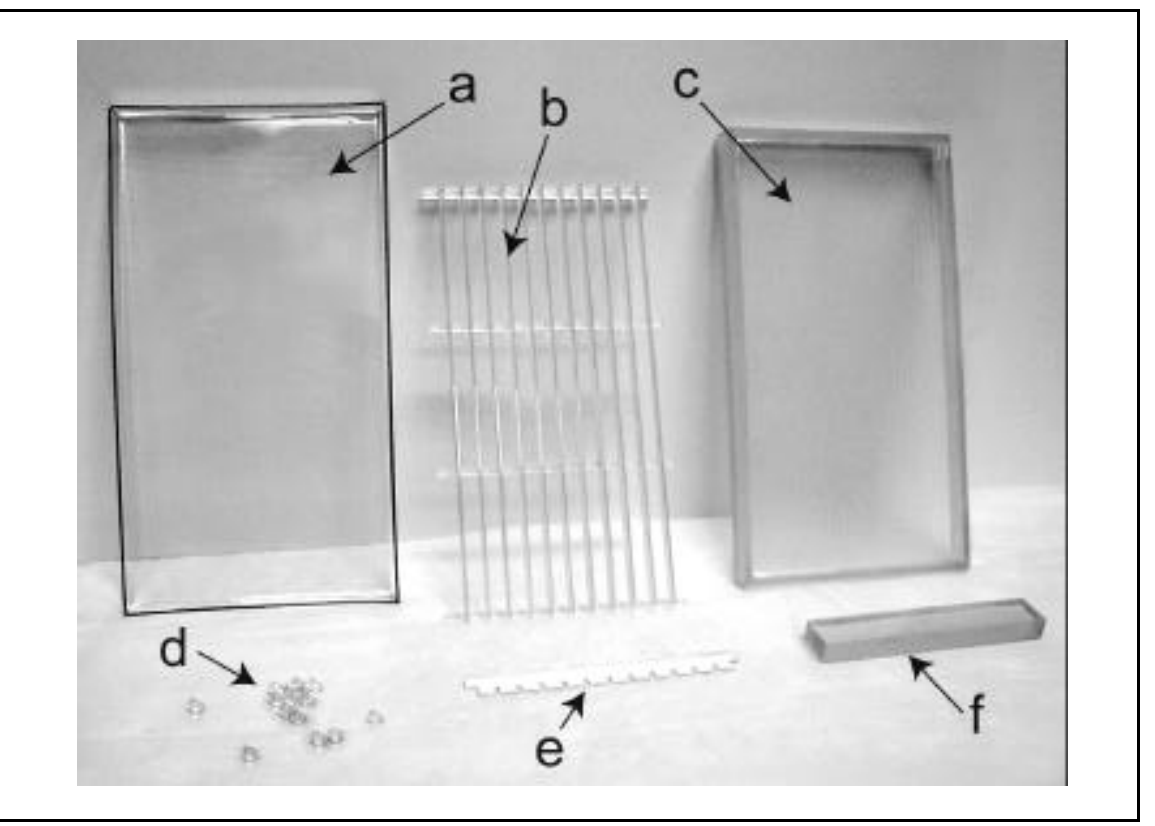

Figure 1. Components of the Multichannel Plating Unit. (a) Lid (formed by heat-folding optically clear polycarbonate of $0.1 \mathrm{~cm}$ thickness). (b) Insert with 11 divider lanes with three cross-supports underneath and notches for the start gate. (c) Bottom tray (with the exit pore cut into the tray edge in the exit alley; see Figure 3). (d) Glass beads (from any commercially available source). (e) Start gate (similar to an agarose gel comb). (f) Exit block (solid block of polycarbonate that fits snugly into the exit alley yet can be easily removed). 


\section{High-Throughput M ethods}

ized before use. The unit shown here is made of autoclavable polycarbonate and is autoclaved fully assembled. That is, the lane divider insert is placed in the tray, the exit block and start gate are inserted, and the unit is covered with the lid. Following sterilization of the unit, the lid is removed and $145 \mathrm{~mL}$ agar-based culture medium is poured into the unit and allowed to solidify. This volume of agar fills the unit such that the start gate rests just above and does not touch the surface of the solidified agar. For transformation of E. coli, we typically used Lennox Agar (Invitrogen, Carlsbad, CA, USA) with antibiotic added (50 mg/L kanamycin) for the selection of transformants. After the medium has solidified, the unit can be stored at $4{ }^{\circ} \mathrm{C}$ until needed. Any medium (suitable for growth of the organism) that can be poured as a liquid and subsequently solidified is suitable. Before use, one sterile glass bead $(0.6 \mathrm{~cm}$ diameter) is added (using sterile technique) to each of the 12 lanes between the start gate and the top of the unit, and the exit block is removed.

\section{Plating Bacterial Samples}

Transformation of $E$. coli can be carried out according to any standard protocol (e.g., Reference 2). For other organisms or cell cultures, any transformation method that is amenable to being carried out in a 96-well format would be suitable. The use of a 12channel pipettor and sterile tips makes it possible to load 12 cultures from a transformation block onto the plating unit at the same time (Figure 2). Typical volumes that work well (i.e., give rise to individual colonies) are 30-50 $\mu \mathrm{L} /$ culture. In an $E$. coli transformation experiment, aliquots of liquid culture are loaded onto the sterile beads in the Multichannel Plating Unit following the $37^{\circ} \mathrm{C}$ incubation/recovery period. Because the volume of culture required to generate single colonies is dependent on the overall efficiency of the cloning experiment, this volume will need to be determined empirically. Next, the unit is gently agitated by knocking the beads back and forth between the start gate and the edge of the unit to coat the beads with the culture. Then, the start gate is removed and the unit is tilted to allow the beads to roll down the agar lanes and to drop into the exit alley. The unit is then tilted toward a corner of the unit so that the beads fall through a pore in the exit alley (Figure 3 ).

When plating out transformants, the goal is to generate single colonies. The simplest method of achieving this is to load the cultures onto the beads and then to roll the beads directly from the start gate into the exit alley. However, if for whatever reason it is desirable to spread the entire volume of media that is loaded onto the beads, then leaving the exit block in place and rolling the beads back and forth in the unit is an

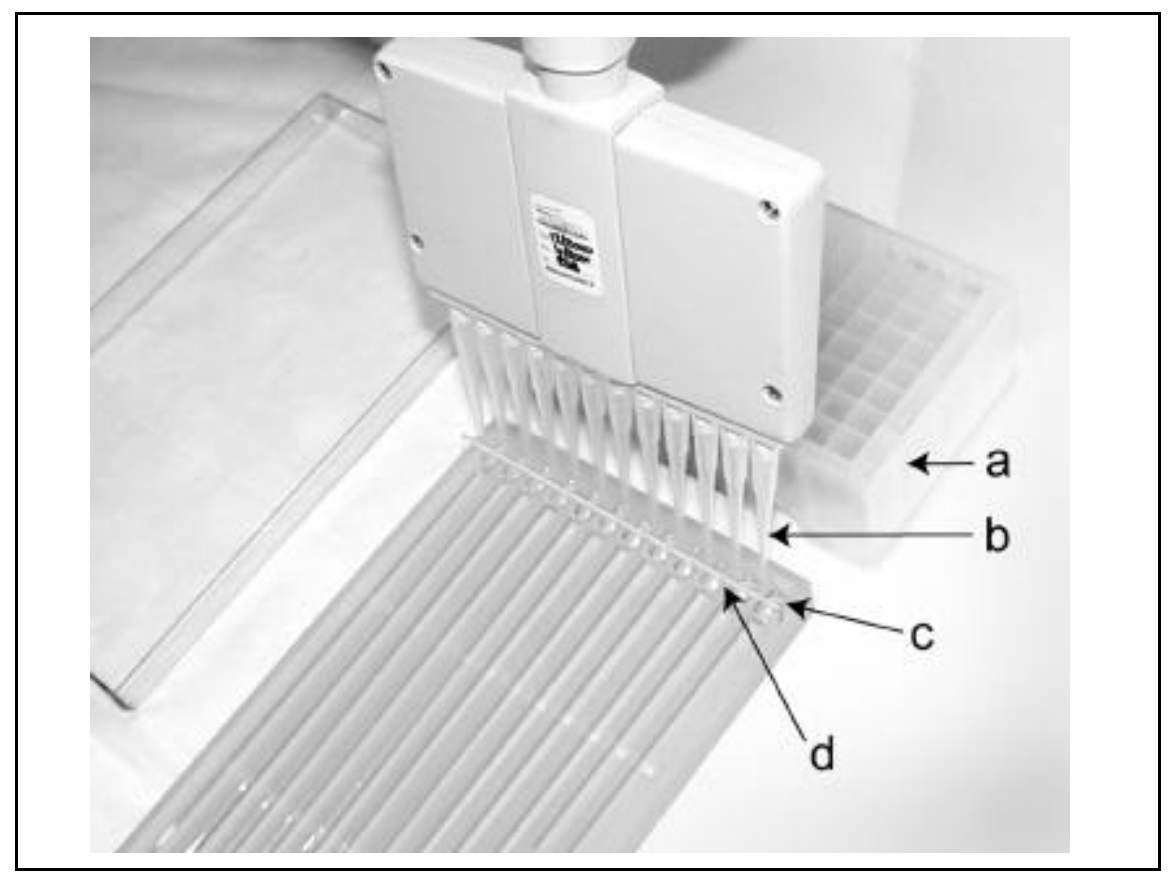

Figure 2. Loading the Multichannel Plating Unit. (a) 96-well culture growth block. (b) 12-channel pipettor with aliquots of culture. (c) Glass beads being loaded with culture. (d) Start gate.

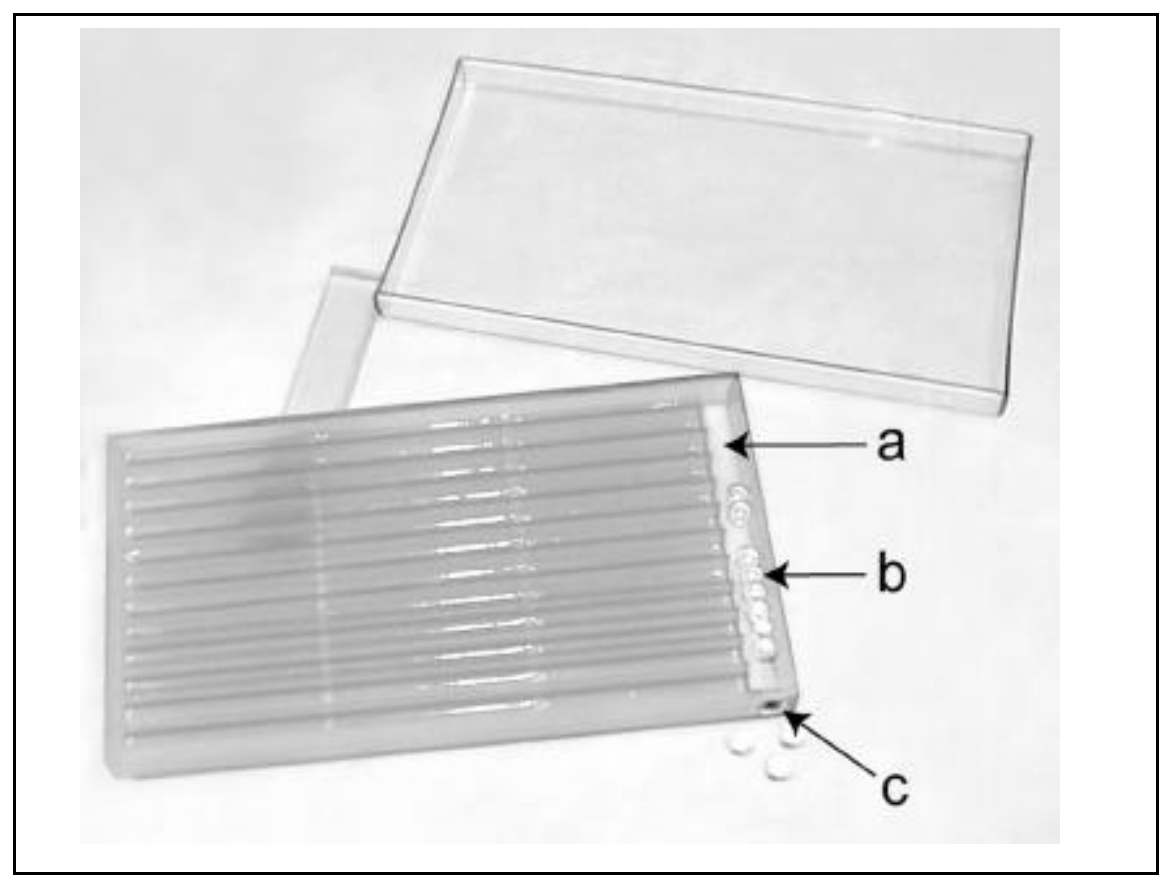

Figure 3. Removal of the beads. (a) Exit alley. (b) Beads in exit alley. (c) Bead exit pore. 
option. In either case, the method can be optimized to generate single colonies by adjusting the volume of culture that is loaded onto the beads.

After the cultures are plated, the unit is left at room temperature until any remaining liquid is absorbed (10-15 $\mathrm{min}$ ). The unit is then inverted (with the lid on) and placed in an appropriate (typically $37^{\circ} \mathrm{C}$ ) incubator. After incubation overnight (at $37^{\circ} \mathrm{C}$ ), single colonies are observed and can be picked using sterile toothpicks. Single colonies from an E. coli transformation experiment are shown in Figure 4.

\section{RESULTS AND DISCUSSION}

The Multichannel Plating Unit provides a more efficient way of plating out bacterial or other types of cell cultures in a high-throughput environment. A skilled technician can plate out 96 transformations in approximately 3 h. That includes getting out the plates, labeling the plates, plating out the cultures, and putting the plates into the incubator. Using a 12-channel pipettor and the Multichannel Plating Unit described here, this time requirement is reduced to approximately $20 \mathrm{~min}$. The time and effort required to pour eight

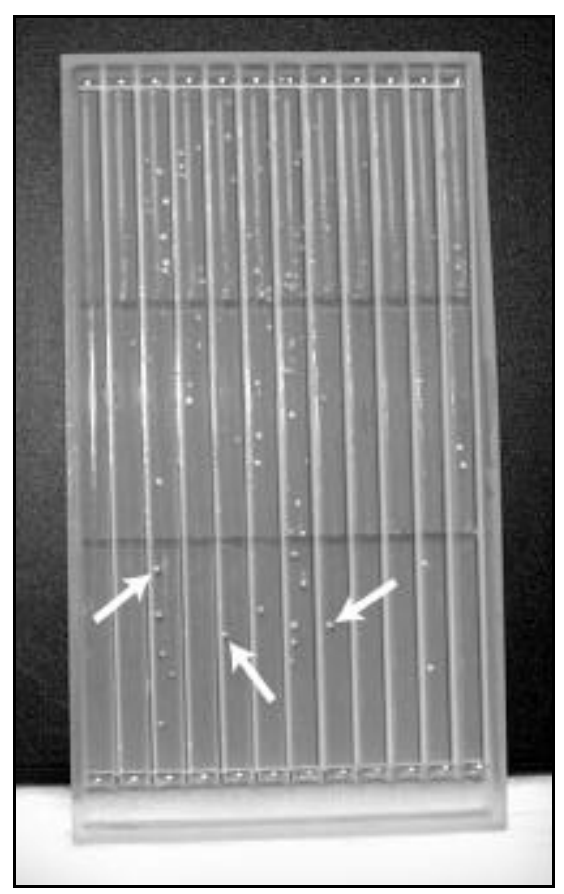

Figure 4. Individual E. coli colonies.
Multichannel Plating Units is less than that required to pour 96 individual petri plates. Disposable petri dishes are no longer used, and the volume of agar required to fill eight Multichannel Plating Units $(145 \mathrm{~mL} \times 8=1160 \mathrm{~mL})$ is reduced compared to 96 individual petri dishes $(20 \mathrm{~mL} \times 96=1920 \mathrm{~mL})$. It is also reasonable to expect labeling and plating errors to be reduced. That is, the current practice is to individually label 96 petri dishes so that each corresponds to a specific location in the culture block (i.e., A1-H12). Then, the cultures are plated out one at a time onto the labeled petri dishes. Plating a culture onto a petri dish with a non-corresponding label is a common error. If the Multichannel Plating Unit is used, then there are only eight plates to label. Because the beads are loaded using a multichannel pipettor, the order of the wells (i.e., A1-A12) is preserved.

Automation of the Multichannel Plating Unit could further reduce the human effort and error inherent in the high-throughput plating of cultures. The most tedious step in the use of the Multichannel Plating Unit is the addition of the sterile beads to the lanes. This step could be facilitated by the development of a simple dispenser for the beads. One can also envision a robotics station with arms that can carry out the following: $(i)$ load the beads, (ii) pipet aliquots of culture from a growth block onto the beads, and (iii) remove the start gate. The station would include a platform to hold the unit that could be agitated to coat the beads and tilted to roll the beads down the agar lanes and out the exit pore. It is conceivable that with multiple platforms eight Multichannel Plating Units could be loaded at the same time, thus effectively plating out an entire 96-well culture block in a matter of minutes. Even without automation, the Multichannel Plating Unit will greatly reduce one of the most tedious, labor-intensive, and error-prone steps remaining in highthroughput molecular biology.

\section{ACKNOWLEDGMENTS}

The authors acknowledge Keith Koutsky of Paradigm Genetics for critical reading of the manuscript and for help with the supplementary drawings.

\section{REFERENCES}

1.Hamilton, C.M. High-throughput method apparatus, and kit for plating microorganisms and cell cultures. US Patent Pending.

2.Hanahan, D. 1983. Studies on transformation of Escherichia coli with plasmids. J. Mol. Biol. 166:557-580.

3.Shibata, K., M. Itoh, K. Aizawa, S. Nagaoka, N. Sasaki, P. Carninci, H. Konno, J. Akiyama, et al. 2000. RIKEN integrated sequence analysis (RISA) system-384-format sequencing pipeline with 384 multicapillary sequencer. Genome Res. 11:1757-1771.

4.Strausberg, R.L., E.A. Feingold, R.D. Klausner, and F.S. Collins. 1999. The mam malian gene collection. Science 286:455-457.

Received 14 March 2002; accepted 2 May 2002.

\author{
Address correspondence to: \\ Dr. Carol M. Hamilton \\ Paradigm Genetics \\ 108 Alexander Drive \\ Research Triangle Park, NC 27709-4528, USA \\ e-mail: chamilton@paragen.com
}

For reprints of this or any other article, contact Reprints@BioTechniques.com 